\title{
A production inventory model with exponential demand rate and reverse logistics
}

\author{
Ritu Raj $^{a^{*}}$, Naresh Kumar Kaliraman ${ }^{a}$, Shalini Chandrab and Harish Chaudhary ${ }^{\mathrm{c}}$
}

${ }^{a}$ Research Scholar, Centre for Mathematical Sciences, Banasthali University, P.O. Banasthali Vidyapith, Rajasthan-304022, India

${ }^{b}$ Associate Professor, Centre for Mathematical Sciences, Banasthali University, P.O. Banasthali Vidyapith, Banasthali - 304022, Rajasthan, India ${ }^{c}$ Assistant Professor, Department of Management Studies, Indian Institute of Technology, Delhi, New Delhi-110016, India

\section{H R O N I C L E}

Article history:

Received January 62014

Received in Revised Format

June 152014

Accepted July 172014

Available online

July 312014

Keywords:

Imperfect Production

Reverse Logistics

Integrated

Supply Chain \begin{abstract}
A B S T R A C T
The objective of this paper is to develop an integrated production inventory model for reworkable items with exponential demand rate. This is a three-layer supply chain model with perspectives of supplier, producer and retailer. Supplier delivers raw material to the producer and finished goods to the retailer. We consider perfect and imperfect quality products, product reliability and reworking of imperfect items. After screening, defective items reworked at a cost just after the regular manufacturing schedule. At the beginning, the manufacturing system starts produce perfect items, after some time the manufacturing system can undergo into "out-of-control" situation from "in-control" situation, which is controlled by reverse logistic technique. This paper deliberates the effects of business strategies like optimum order size of raw material, exponential demand rate, production rate is demand dependent, idle times and reverse logistics for an integrated marketing system. Mathematica is used to develop the optimal solution of production rate and raw material order for maximum expected average profit. A numerical example and sensitivity analysis is illustrated to validate the model.
\end{abstract}

(O) 2014 Growing Science Ltd. All rights reserved

\section{Introduction}

Mathematical modeling, analysis and calculation are important areas for research in the study of supply chain system. Supplier, producer and retailer are the members of three-layer supply chain. Supplier supplies the raw material to producer and finished goods to retailers and customers. The manufacturing process shift to out-of-control state from in-control state after some time and produced some imperfect objects. These imperfect objects reworked just after the regular manufacturing schedule. The combination among suppliers, producers and retailers is important for an integrated supply chain system. Rosenblatt and Lee (1986) considered a reverse logistic model where the probability distribution of the instant of changing from in-control situation to out-of-control situation follows an exponential distribution. They understood that the imperfect objects produced in out-of-control situation could be reworked immediately at a price and establish that the presence of imperfect products outcome in a minor set. On the beginning of reverse logistic model, Lee and Rosenblatt (1987) showed that process analysis through the manufacturing runtime could perceive the changing time and it might be restored previously.

* Corresponding author.

E-mail: rrituiit@gmail.com (R.Raj)

(C) 2014 Growing Science Ltd. All rights reserved.

doi: $10.5267 /$ j.ijiec. 2014.7 .006 
Liu and Yang (1996) investigated a particular stage manufacturing setup with defective method of supplying of reworkable and non-reworkable objects. The impact of imperfect objects on the batch sizing procedure was renowned in the works of Sana et al. (2007, 2007a), with others. Kim and Hong (1999) considered that imperfect objects could be found after the manufacturing run and the imperfect objects were reworked at a rate. Banerjee and Kim (1995) considered the integrated inventory models in which the retailer and customer coordinate their manufacturing and ordering plan, in order to lesser the common inventory costs.

Khouja and Mehrez (1994) considered three coordination systems among the members of the supply chain and showed that several coordination systems could induce to significant decrease in total price. Cardenas-Barron (2007) extended the model of Khouja and Mehrez (1994) by mathematical method, assuming n-stage multi-customer supply chain inventory coordination. Jalbar et al. (2008) developed a multi-echelon inventory coordination in which one retailer delivers an item to many customers. Chiu (2003) developed an economic production quantity model with backorders by combining the assumptions of a symmetrical of the imperfect objects were reworked to build them superior quality objects instead of reworking on all the imperfect objects and the remaining objects are sold at a low rate. Yang and Wee (2001) developed a supply chain model by integrating producer, distributer and vendor as three members of the chain.

Cardenas-Barron (2008) presented a straightforward derivation to discover best production lot size with rework procedure at solitary phase manufacturing method. Cardenas-Barron (2009) developed an economic production quantity model with planned backorders for determining the manufacturing batch size and the size of backorders in an imperfect production process where all imperfect objects were reworked at the similar rotation. Sana and Chaudhuri (2010), Sana (2010, 2010a), Sarkar et al. (2010) and Chiu et al. (2007) showed that the imperfect objects might be reworked at a cost where overall production-inventory costs could be decreased considerably.

Chiu et al. (2007) developed the job of Chiu (2003) and considered the best run-time difficulty of economic production quantity model with scrap, reworking of imperfect objects and stochastic breakdowns. Sarker et al. (2008) addressed the problem connecting to reworking of imperfect objects in a multi-stage manufacturing method by considering two operational policies: reworking of imperfect objects within the same cycle and after $N$ cycles. Biswas and Sarker (2008) described an inventory system of a solitary manufacturing procedure with an in-cycle rework strategy of fragment with complete analysis. Sana (2011) developed an integrated production inventory model of perfect and imperfect quality products in a three-layer supply chain.

Jamal et al. (2004) developed a manufacturing system with rework process consisting two cases of rework process to minimize the total production cost. At the first instant, they executed reworking in the same cycle. At the second instant, they executed reworking after $N$ cycles. Chiu et al. (2007) considered a manufacturing system with rework, including optimal lot sizing decision, random scrap rate and service level constraint. They derived that the expected total cost of a manufacturing system is less if backlogging is allowed or equal if backlogging is not allowed. Hafshejani et al. (2012) presented a multi-product economic production quantity model with imperfect quality items and reworking, it is a nonlinear programming problem solved by genetic algorithm with limited warehouse space.

Krishnamoorthi and Panayappam (2013) considered a single stage manufacturing system in which imperfect quality items produced and reworked. They developed two production models, one with shortages and second without shortages. Pasandideh et al. (2010) considered a multi-product economic production quantity model with defective products, reworking and limited warehouse space. Liao et al. (2009) examined an integrated repairable and manufacturing procedure with economic production quantity model with defective maintenance and rework upon fulfillment. Haji et al. (2009) developed a model for an inventory problem considering a single machine with imperfect quality items. They considered that all imperfect quality items are to be reworked, setup cost for rework, no shortages are 
allowed and waiting time for imperfect quality item. Peter Chiu et al. (2010) developed a mathematical model to establish the optimal run time for a defective fixed production rate with rework, scrap and stochastic machine wreck. Wee and Chung (2009) and Chung and Wee (2010) developed an integrated production inventory model with deterioration assuming rework and green component value design. They extended the economic production quantity model with product reliability. We developed a mathematical model of three-layer supply chain including supplier, producer and retailer. The imperfect items at retailer's level are sent back to the external supplier and the imperfect items at producer's level are reworked after regular manufacturing time. The average profit of producer at incontrol situation and out-of-control situation is considered to derive the solution of the problem. The average profits of suppliers and retailers are considered also.

\section{Essential assumptions and symbolizations}

The mathematical model is developed on the bases of following assumptions:

1. The demand rates are exponential increasing function of time for each member of three layer supply chain.

2. Production rate is demand dependent i.e. $P(t)=\lambda D(t)$, where $D(t)=b e^{a t}$ and $\lambda>1$

3. Production cost per unit item is production rate dependent.

4. Idle time costs are assumed at supplier's and producer's level.

5. Different probability distributions functions are considered for defective items at supplier's level.

6. Single item products are considered for joint effect of supplier, producer and retailer in a three layer supply chain.

7. Replenishment rate is instantly infinite but its size is finite at supplier's level.

8. Stock-out situation is not conceded.

9. No product of producer is discarded at any stage.

10. No imperfect items are manufactured during rework.

11. Reworking starts just after the regular production process in each production run.

12. The imperfect objects are restored to its original quality, after reworking.

13. Insignificant lead time.

The mathematical model is developed on the bases of following symbolizations:

$Q_{s}(t)$ Supplier's on-hand inventory of good items at time $t$,

$Q_{p}(t)$ Producer's on-hand inventory of good items at time $t$,

$Q_{p_{r}}(t)$ Producer's on-hand inventory of defective items which would be reworked, 
$Q_{r}(t)$ Retailer's on-hand inventory of good items at time $t$,

$R \quad$ Supplier's replenishment lot size,

$\lambda b e^{a t} \quad$ Producer's production rate that is equal to supplier's demand rate,

$\alpha \quad$ Supplier's proportional probability of imperfect items with probability density function $f(\alpha)$,

$A_{s} \quad$ Supplier's set up cost,

$r_{s} \quad$ Supplier's screening rate per unit time,

$S_{c} \quad$ Supplier's screening cost per unit item,

$h_{s} \quad$ Supplier's holding cost per unit per unit time,

$I_{s} \quad$ Supplier's cost per unit idle time,

$C_{s} \quad$ Supplier's purchasing cost per unit item,

$w_{s} \quad$ Supplier's selling price per unit perfect items,

$\overline{w_{s}} \quad$ Supplier's selling price per unit imperfect items,

$E(x)$ Expected value of variable $x$,

$S A P \quad$ Supplier's average profit,

ESAP Supplier's expected average profit,

$\beta \quad$ The percentage of imperfect items in "out-of-control" situation at producer level,

$\lambda b e_{1}^{a t} \quad$ Rate of reworking per unit time,

$\tau \quad$ Random time with mean $\frac{1}{\theta}$ after which the production system becomes uncontrollable for producer,

$F(\tau) \quad$ Probability distribution function of $\tau$,

$f(\tau) \quad$ Probability density function of $\tau$,

$A_{p} \quad$ Producer's set up cost,

$r_{p} \quad$ Producer's screening rate per unit time,

$S_{p} \quad$ Producer's screening cost per unit item,

$h_{p} \quad$ Producer's holding cost per unit per unit time for perfect items,

$h_{p}{ }^{\prime} \quad$ Producer's holding cost per unit per unit time for defective items, which would be reworked,

$r_{p}^{\prime} \quad$ Cost to rework for imperfect item of producer,

$L \quad$ Cost of labor, energy, technology at fixed rate,

$\gamma \quad$ Cost of tool and die is a variation constant,

$p_{I} \quad$ Producer's cost per unit idle time,

$C(P) \quad$ Per unit item production cost,

$N \quad$ Numbers of imperfect objects in the production process,

$w_{p} \quad$ Producer's selling price per unit perfect item,

$\overline{w_{p}} \quad$ Producer's selling price per unit imperfect item,

$P A P \quad$ Producer's average profit,

EPAP Producer's expected average profit,

$b e_{c}^{a t} \quad$ Customer's demand rate,

$b e_{r}{ }^{a t} \quad$ Retailer's demand rate, 
$A_{r} \quad$ Retailer's set up cost,

$h_{r} \quad$ Retailer's holding cost per unit per unit time,

$w_{r} \quad$ Retailer's selling price per unit item,

$R A P \quad$ Retailer's average profit,

ERAP Retailer's expected average profit,

T Retailer's cycle length.

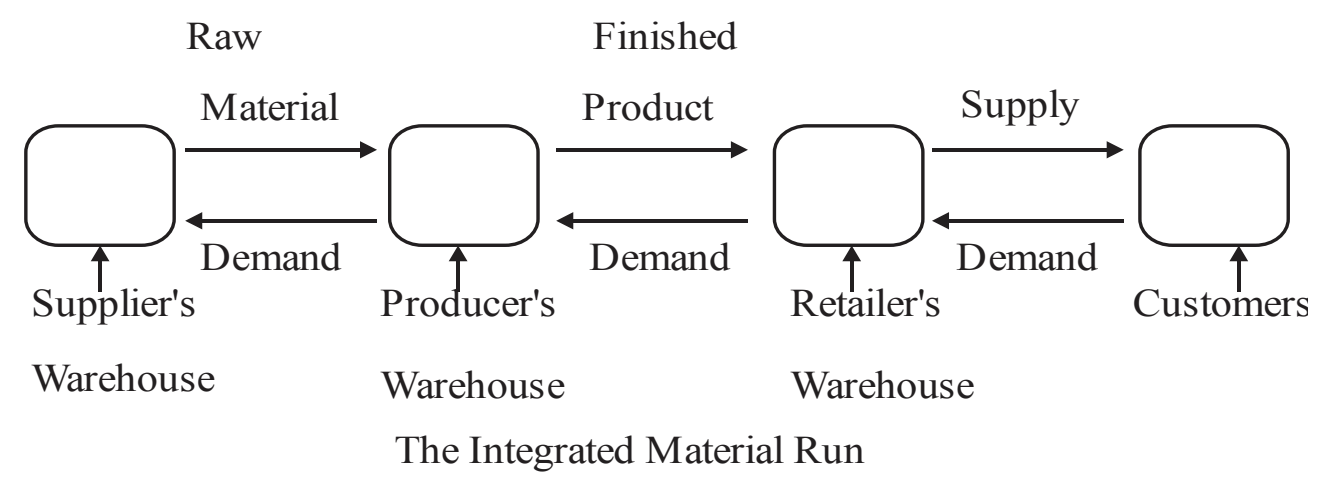

Fig. 1. The integrated material run

\section{Mathematical Model}

In this projected model, supplier delivers the raw materials at rate $\lambda b e^{a t}$ to the producer up to manufacturing run time $t_{1}$. The imperfect objects at supplier level are sent back after examine carefully with sale rate $\overline{w_{s}}$ per unit item to the external dealer from where the raw materials are purchased. We assumed that the manufacturing process is "in-control" state at starting stage. The process may shift to an "out-off-control" state and may produce imperfect objects after a random time $(\tau)$ with mean $\left(\frac{1}{\theta}\right)$. The total imperfect objects manufactured at time $t_{1}$ are reworked with production rate $\lambda b e_{1}^{a t}$, which takes time $t_{r}$. The inventory fills up after accommodating the demand $b e_{r}{ }^{a t}$ of retailer during manufacturing run time $t_{1}$. The customers demand is fulfilled with rate $b e_{c}^{a t}$ by retailer where the delivery charge of produced items is sustained up to time $k T(k<1)$. The assembled inventory at time $k T$ decreases and accomplishes to zero level at time $T$. The leading differential equations at supplier, producer and retailer level are as follows:

\subsection{Supplier's specific average profit}

The leading differential equation is

$$
\frac{d Q_{s}(t)}{d t}=-\lambda b e^{a t}
$$

with $Q_{S}(0)=(1-\alpha) R$ and $Q_{S}\left(t_{1}\right)=0, \quad 0 \leq t \leq t_{1}$

From Eq. (1), we have 
$Q_{s}(t)=\frac{\lambda b}{a}\left(1-e^{a t}\right)+(1-\alpha) R, 0 \leq t \leq t_{1}$

We have $Q_{S}\left(t_{1}\right)=0$

$\Rightarrow t_{1}=\frac{1}{a} \log \left(1+\frac{a(1-\alpha) R}{\lambda b}\right)$

The inventory cost of perfect objects is

$I C=\frac{h_{s}}{a}\left[\left(\frac{\lambda b}{a}+(1-\alpha) R\right) \log \left(1+\frac{a(1-\alpha) R}{\lambda b}\right)-(1-\alpha) R\right]$

Inventory cost of defective objects is

$I C_{i}=\frac{h_{S} \alpha R^{2}}{r_{s}}$

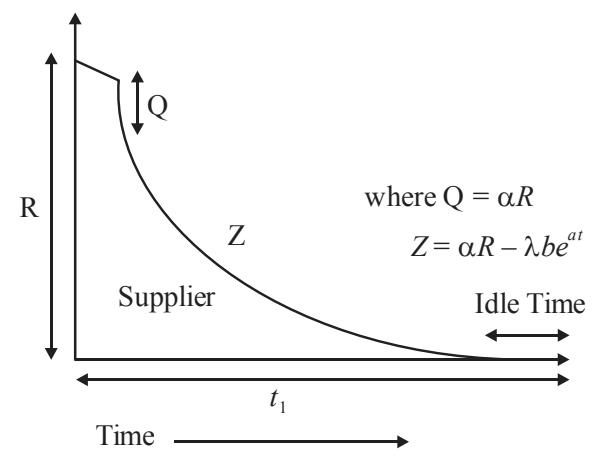

Fig. 2. Trend on inventory change

The price of screening is $S_{c} R$. The earning from selling the perfect and imperfect items is $\left(w_{s}(1-\alpha) R+\overline{w_{s}} \alpha R\right)$. The buying price of $R$ objects is $C_{s} R$. The price of establishment is $A_{s}$. The idle time cost is $I_{s}\left(T-t_{1}\right)$. The supplier's average profit is, using $t_{1}=\frac{1}{a} \log \left(1+\frac{a(1-\alpha) R}{\lambda b}\right)$

$$
\begin{aligned}
S A P & =\frac{1}{T}\left[w_{s}(1-\alpha) R+\overline{w_{s}} \alpha R-\left(S_{c}+C_{s}\right) R-I_{s}\left(T-\frac{1}{a} \log \left(1+\frac{a(1-\alpha) R}{\lambda b}\right)\right)\right] \\
& -\frac{1}{T}\left[\frac{h_{s}}{a}\left\{\left(\frac{\lambda b}{a}+(1-\alpha) R\right) \log \left(1+\frac{a(1-\alpha) R}{\lambda b}\right)-(1-\alpha) R+\frac{a \alpha R^{2}}{r_{s}}\right\}+A_{s}\right]
\end{aligned}
$$

\subsection{Producer's specific average profit}

If a manufacturing process does not perform well according to process designer's specification then it is said to be "out-of-control". The constraint $\theta$ is a design variable and an indicator of manufactured goods consistency. Consistency of machines in a production system is usually authenticated to be an exponential function of time $t$ which is $R(t)=e^{-\theta t}$, where $\theta=\frac{\text { imperfect digits }}{\text { total number of working hours }}$. Therefore, a unit either fails or carries on and one of these two states surrogates must exist. We have $R(t)=1-F(t)$. 
Here $F(t)=\int_{0}^{t} f(u) d u$, where $f(t)$ is the failure probability density function then $\int_{0}^{\infty} f(t) d t=1$. Thus $f(t)=\frac{d}{d t}(F(t))=\theta e^{-\theta t}$

The unit production cost is

$C(P)=w_{s}+\frac{L}{\lambda b e^{a t}}+\gamma \lambda b e^{a t}$

We assumed that the manufacturing process is in "in-control" state at starting stage. The process may shift to an "out-off-control" state and may produce imperfect objects after a random time $(\tau)$ with mean $\left(\frac{1}{\theta}\right)$. The holding cost per unit per unit time for defective items is greater than the holding cost per unit per unit time for perfect items. Two cases are gets up in this operating system:

3.2.1 Case I: When $0 \leq \tau \leq t_{1}$, the "out-of-control" state exists during operations time. At the beginning, the production rate is $\lambda b e^{a t}$. The inventory fills up after accommodating the demand $b e_{r}{ }^{a t}$ of retailer during manufacturing run time $t_{1}$. Thus $(\tau)$ exists in the time period $\left(0, t_{1}\right)$, the perfect items are produces during $[0, \tau]$ and it produces both perfect and imperfect items during $\left[\tau, t_{1}\right]$. The total numbers of items manufactured during $\left(0, t_{1}\right)$ are used to fulfill the demand for the time duration $\left(t_{1}+t_{2}\right)$. Since, the total numbers of imperfect objects manufactured at time $t_{1}$ are reworked with manufacturing rate $\lambda b e_{1}^{a t}$ which takes time $t_{r}$. The time $t_{3}$ is necessary to sell out the reworked items. On-hand inventory of perfect items at time $t$ can be illustrated by the following differential equations:

$\frac{d Q_{p}(t)}{d t}=\lambda b e^{a t}-b e_{r}^{a t}$

with $Q_{p}(0)=0,0 \leq t \leq \tau$

$\frac{d Q_{p}(t)}{d t}=(1-\beta) \lambda b e^{a t}-b e_{r}^{a t}$

with $Q_{p}(\tau)=\left(\lambda b e^{a t}-b e_{r}{ }^{a t}\right) \tau, \tau \leq t \leq t_{1}$

$\frac{d Q_{p}(t)}{d t}=-b e_{r}^{a t}$

with $Q_{p}(0)=Q_{p}\left(t_{1}\right)$ and $Q_{p}\left(t_{2}\right)=0,0 \leq t \leq t_{2}$

From Eq. (8), we have

$Q_{p}(t)=\left\{\frac{b}{a}\left(1-e_{r}^{a t}\right)-\frac{\lambda b}{a}\left(1-e^{a t}\right)\right\}, 0 \leq t \leq \tau$

From Eq. (9), we have 
$Q_{p}(t)=b\left(\lambda e^{a t}-e_{r}^{a t}\right) \tau+\frac{(1-\beta) \lambda b}{a}\left(e^{a t}-e^{a \tau}\right)+\frac{b}{a}\left(e_{r}^{a \tau}-e_{r}^{a t}\right), \tau \leq t \leq t_{1}$

From Eq. (10), we have

$Q_{p}(t)=b\left(\lambda e^{a t_{1}}-e_{r}^{a t_{1}}\right) \tau+\frac{(1-\beta) \lambda b}{a}\left(e^{a t_{1}}-e^{a \tau}\right)+\frac{b}{a}\left(e_{r}^{a t}+e_{r}^{a \tau}-e_{r}^{a t_{1}}-1\right), 0 \leq t \leq t_{2}$

Now, $Q_{p}\left(t_{2}\right)=0$

We have

$t_{2}=\frac{1}{a} \log \left[(a \tau+1) e_{r}^{a t_{1}}-(a \tau \lambda+(1-\beta) \lambda) e^{a t_{1}}+(1-\beta) \lambda e^{a \tau}-e_{r}^{a \tau}+1\right]$

Using $t_{1}=\frac{1}{a} \log \left(1+\frac{a(1-\alpha) R}{\lambda b}\right)$, we have

$t_{2}=\frac{1}{a} \log \left[\{(a \tau+1)-\lambda(a \tau+1-\beta)\}\left(1+\frac{a(1-\alpha) R}{\lambda b}\right)+(1-\beta) \lambda e^{a \tau}-e_{r}^{a \tau}+1\right]$

The inventory level $Q_{r}(t)$ of defective items at time $t$ liquidates the following differential equations:

$\frac{d Q_{p}(t)}{d t}=\beta \lambda b e^{a t}$

with $Q_{p}(\tau)=0, \tau \leq t \leq t_{1}$

$\frac{d Q_{p}(t)}{d t}=-b e_{r}^{a t}$

with $Q_{p}(0)=Q_{p}\left(t_{1}\right)=\beta \lambda b e^{a t}\left(t_{1}-\tau\right)$ and $Q_{p}\left(t_{3}\right)=0,0 \leq t \leq t_{3}$

From Eq. (15a) and Eq. (15b), we have

$Q_{p}(t)=\frac{\beta \lambda b}{a}\left(e^{a t_{1}}-e^{a \tau}\right), \tau \leq t \leq t_{1}$
$Q_{p}(t)=\frac{\beta \lambda b}{a}\left(e^{a t_{1}}-e^{a \tau}\right)-\frac{b}{a}\left(1-e_{r}^{a t}\right), 0 \leq t \leq t_{3}$

Thus, $Q_{p}\left(t_{3}\right)=0$

$t_{3}=\frac{1}{a} \log \left[1-\beta \lambda\left(1+\frac{a(1-\alpha) R}{\lambda b}-e^{a \tau}\right)\right]$ 
Here, $k T=t_{1}+t_{2}+t_{3}$

$$
k T=\frac{1}{a} \log \left[\begin{array}{l}
\left(1+\frac{a(1-\alpha) R}{\lambda b}\right)\left\{\begin{array}{l}
(a \tau+1)\left(1+\frac{a(1-\alpha) R}{\lambda b}\right)-\lambda(a \tau+1-\beta) \\
\left(1+\frac{a(1-\alpha) R}{\lambda b}\right)+(1-\beta) \lambda e^{a \tau}-e_{r}^{a \tau}+1
\end{array}\right\} \\
\left\{1-\beta \lambda\left(1+\frac{a(1-\alpha) R}{\lambda b}-e^{a \tau}\right)\right\}
\end{array}\right]
$$

Thus, the inventory cost for perfect objects is

$$
H G_{p}=h_{p}\left[\begin{array}{l}
\frac{b}{a^{2}}\left(a t_{1} e_{r}^{a \tau}-e_{r}^{a t_{1}}-a \tau e_{r}^{a \tau}+a \tau-\lambda\left(1-e^{a \tau}+a \tau\right)\right)+\frac{b \tau}{a}\left(\lambda e^{a t_{1}}-e_{r}^{a t_{1}}-\lambda e^{a \tau}+e_{r}^{a \tau}\right) \\
+\frac{(1-\beta) \lambda b}{a^{2}}\left(e^{a t_{1}}-a t_{1} e^{a \tau}-e^{a \tau}+\tau e^{a \tau}+a t_{2} e^{a t_{1}}-a t_{2} e^{a \tau}\right)+b\left(\lambda e^{a t_{1}}-e_{r}^{a t_{1}}\right) \tau t_{2} \\
+\frac{b}{a}\left(\frac{1}{a} e_{r}^{a t_{2}}+e_{r}^{a \tau} t_{2}-e_{r}^{a t_{1}} t_{2}-t_{2}\right)
\end{array}\right]
$$

Inventory cost of defective objects is

$$
\begin{aligned}
& H G_{p}^{\prime}=h_{p}^{\prime} {\left[\frac{\beta \lambda b}{a^{2}}\left(e^{a t_{1}}-e^{a \tau}+a \tau e^{a \tau}-a t_{1} e^{a t_{1}}+a t_{r} e^{a t_{1}}-a t_{r} e^{a \tau}\right)+\frac{\lambda b}{a^{2}}\left(e_{1}^{a t_{r}}-a t_{r}-1\right)\right] } \\
&+h_{p}\left[\begin{array}{l}
\left.\frac{b}{a^{2}}\left(e_{r}^{a t_{3}}-1+\lambda-\lambda e_{1}^{a t_{r}}\right)+\frac{b}{a}\left(\lambda t_{r}-t_{3}\right)+\beta \lambda b e^{a t}\left(t_{1}-\tau\right)\left(t_{2}-t_{r}\right)\right] \\
+\frac{\beta \lambda b}{a}\left(e^{a t_{1}}-e^{a \tau}\right) t_{3}
\end{array}\right]
\end{aligned}
$$

Adding Eq. (20) and Eq. (21), the total inventory cost is

$$
\begin{aligned}
T I C=h_{p} \frac{b}{a^{2}}\left[\begin{array}{l}
\left.a\left(\lambda e^{a t_{1}}-e_{r}^{a t_{1}}-\lambda e^{a \tau}+e_{r}^{a \tau}\right) \tau+(1-\beta) \lambda\left(e^{a t_{1}}-e^{a \tau}\right)-\lambda\left(1-e^{a \tau}\right)\right] \\
-e_{r}^{a t_{1}}+e_{r}^{a t_{2}}
\end{array}\right] \\
+h_{p}^{\prime}\left[\frac{\beta \lambda b}{a^{2}}\left(e^{a t_{1}}-e^{a \tau}+a \tau e^{a \tau}-a t_{1} e^{a t_{1}}+a t_{r} e^{a t_{1}}-a t_{r} e^{a \tau}\right)+\frac{\lambda b}{a^{2}}\left(e_{1}^{a t_{r}}-a t_{r}-1\right)\right] \\
+h_{p}\left[\frac{b}{a^{2}}\left(e_{r}^{a t_{3}}-1+\lambda-\lambda e_{1}^{a t_{r}}\right)+\frac{b}{a}\left(\lambda t_{r}-t_{3}\right)+\beta \lambda b e^{a t}\left(t_{1}-\tau\right)\left(t_{2}-t_{r}\right)+\frac{\beta \lambda b}{a}\left(e^{a t_{1}}-e^{a \tau}\right) t_{3}\right]
\end{aligned}
$$

Total expected inventory cost is 


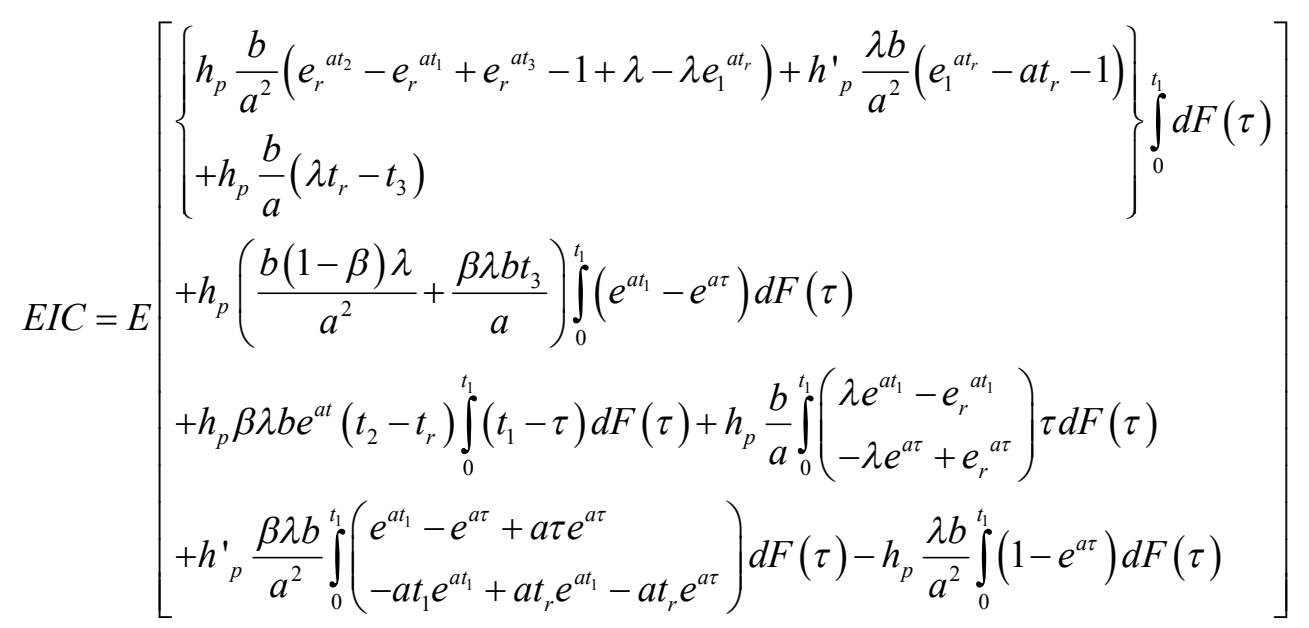

Thus, TIC is a function of random variable $\tau$ and $t_{1}$ depends on random variable $\alpha$. The expected number of imperfect objects in a manufacturing lot size $(1-\alpha) R$ is as follows,

$E(N)=\beta \lambda b e^{a t} \int_{0}^{t_{1}}\left(t_{1}-\tau\right) d F(\tau)=\beta \theta \lambda b e^{a t} \int_{0}^{t_{1}}\left(t_{1}-\tau\right) e^{-\theta \tau} d \tau=\beta \theta \lambda b e^{a t}\left(\frac{t_{1}}{\theta}+\frac{1}{\theta^{2}} e^{-\theta t_{1}}-\frac{1}{\theta^{2}}\right)$

Expand the function $e^{-\theta t_{1}}$ up to third term, we have

$E(N) \approx \frac{1}{2} \beta \theta \lambda b e^{a t} t_{1}^{2}$

Total expected rework cost is

$r_{p} E(N)=\frac{1}{2} r_{p} \beta \theta \lambda b e^{a t} t_{1}^{2}$

The cost of arrangement is $A_{p}$

The income from selling the perfect and imperfect items is

$$
\left\{w_{p}(1-\beta) \lambda b e^{a t_{1}}+\overline{w_{s}} \beta \lambda b e^{a t_{1}}\right\}=\left\{w_{p}+\overline{w_{s}} \frac{\beta}{1-\beta}\right\} b\left\{\lambda(1-\beta)-1+e_{r}^{a k T}\right\}
$$

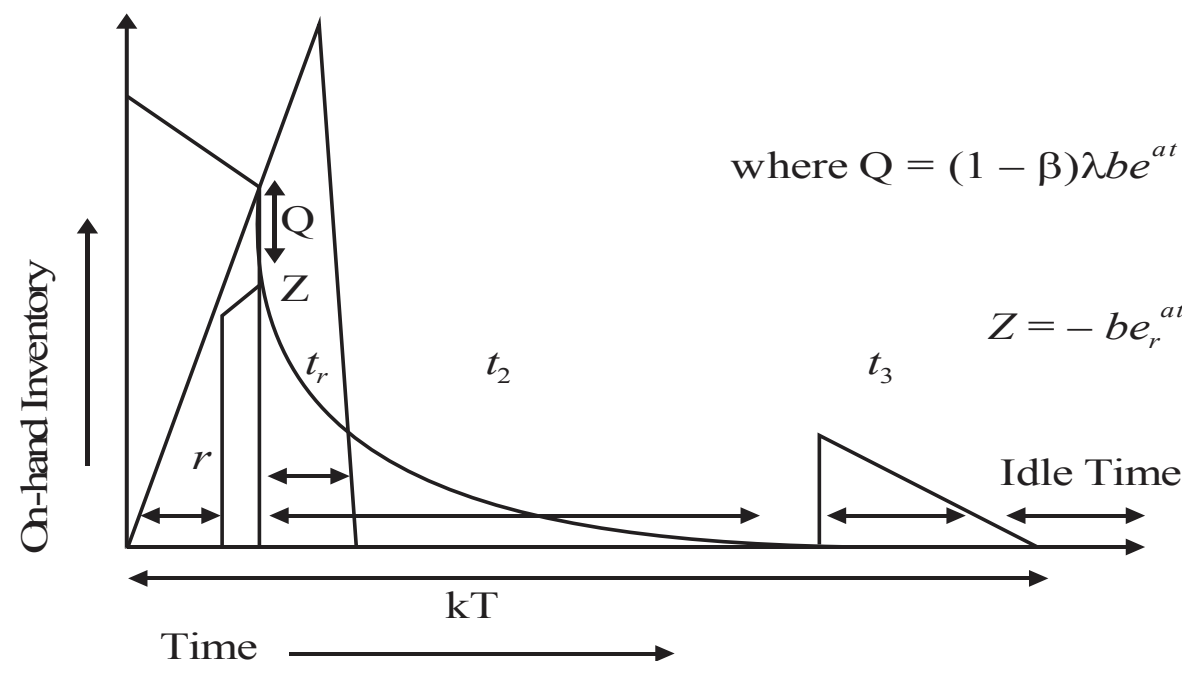

Fig. 3. The change in inventory 
The cost of screening is $S_{p} \lambda b e^{a t_{1}}=\frac{1}{1-\beta} S_{p} b\left\{\lambda(1-\beta)-1+e_{r}^{a k T}\right\}$

In this case,

Total expected profit $=$

$$
E\left[\begin{array}{l}
\left\{w_{p}+\overline{w_{s}} \frac{\beta}{1-\beta}\right\} b\left\{\lambda(1-\beta)-1+e_{r}^{a k T}\right\} \int_{0}^{t_{1}} d F(\tau)-p_{I}\left\{(T-k T)+A_{p}\right\} \int_{0}^{t_{1}} d F(\tau) \\
-\left\{h_{p} \frac{b}{a^{2}}\left(e_{r}^{a t_{2}}-e_{r}^{a t_{1}}+e_{r}^{a t_{3}}-1+\lambda-\lambda e_{1}^{a t_{r}}\right)+h_{p}^{\prime} \frac{\lambda b}{a^{2}}\left(e_{1}^{a t_{r}}-a t_{r}-1\right)+h_{p} \frac{b}{a}\left(\lambda t_{r}-t_{3}\right)\right\} \\
\int_{0}^{t_{1}} d F(\tau)+h_{p} \frac{\lambda b}{a^{2}} \int_{0}^{t_{1}}\left(1-e^{a \tau}\right) d F(\tau)-h_{p}\left(\frac{b(1-\beta) \lambda}{a^{2}}+\frac{\beta \lambda b t_{3}}{a}\right)_{0}^{t_{1}}\left(e^{a t_{1}}-e^{a \tau}\right) d F(\tau) \\
-h_{p} \beta \lambda b e^{a t}\left(t_{2}-t_{r}\right) \int_{0}^{t_{1}}\left(t_{1}-\tau\right) d F(\tau)-h_{p} \frac{b}{a} \int_{0}^{t_{1}}\left(\lambda e^{a t_{1}}-e_{r}^{a t_{1}}-\lambda e^{a \tau}+e_{r}^{a \tau}\right) \tau d F(\tau) \\
-\frac{h_{p}^{\prime} \beta \lambda b^{t_{1}}}{a^{2}} \int_{0}^{t_{1}}\left\{\left(a \tau-a t_{r}-1\right) e^{a \tau}+\left(1-a t_{1}+a t_{r}\right) e^{a t_{1}}\right\} d F(\tau)-r_{p} \beta \lambda b e^{a t} \int_{0}^{t_{1}}\left(t_{1}-\tau\right) d F(\tau)
\end{array}\right]
$$

\subsubsection{Case II:}

When $t_{1} \leq \tau \leq \infty$, the system is in "in-control" state during manufacturing process The leading differential equations are as follows:

$\frac{d Q_{p}(t)}{d t}=\lambda b e^{a t}-b e_{r}^{a t}$

with $Q_{p}(0)=0,0 \leq t \leq t_{1}$

$\frac{d Q_{p}(t)}{d t}=-b e_{r}^{a t}$

with $Q_{p}\left(t^{\prime}{ }_{2}\right)=0$ and $Q_{p}(0)=\left(\lambda b e^{a t}-b e_{r}^{a t}\right) t_{1}, 0 \leq t \leq t^{\prime}{ }_{2}$

From Eq. (25) and Eq. (26), we get

$$
\begin{aligned}
& Q_{p}(t)=\left\{\frac{b}{a}\left(1-e_{r}^{a t}\right)-\frac{\lambda b}{a}\left(1-e^{a t}\right)\right\}, 0 \leq t \leq t_{1} \\
& Q_{p}(t)=\frac{b}{a}\left(1-e_{r}^{a t_{1}}\right)-\frac{\lambda b}{a}\left(1-e^{a t_{1}}\right)+\frac{b}{a}\left(1-e_{r}^{a t}\right), 0 \leq t \leq t^{\prime}{ }_{2}
\end{aligned}
$$

Thus, $Q_{p}\left(t^{\prime}{ }_{2}\right)=0$

$t^{\prime}{ }_{2}=\frac{1}{a} \log \left[2-e_{r}^{a t_{1}}-\lambda\left(1-e^{a t_{1}}\right)\right]$ 


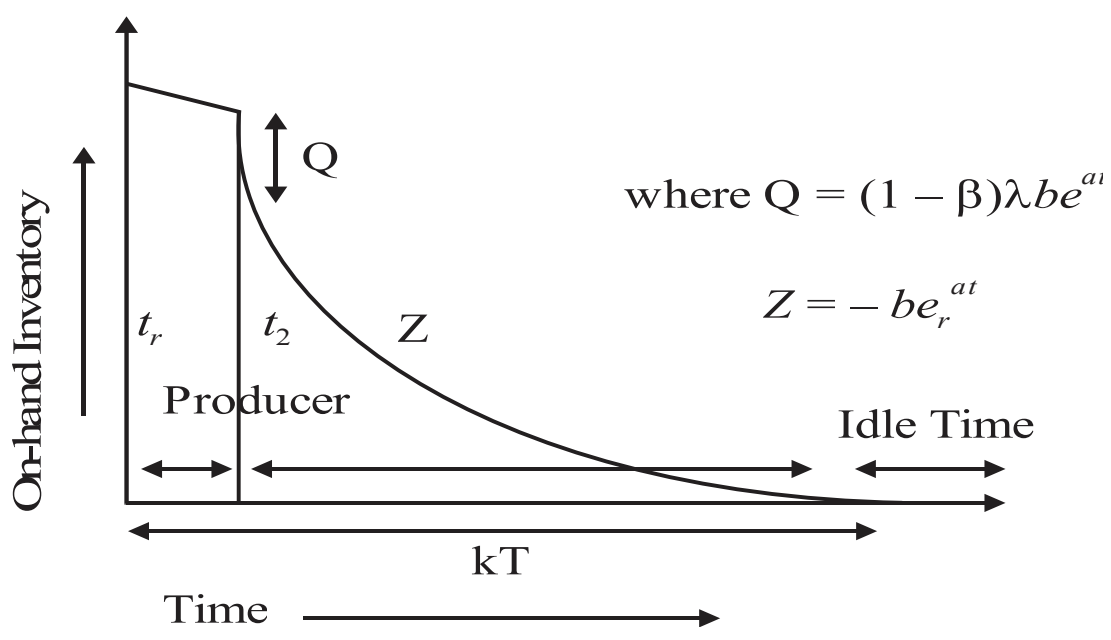

Fig. 4. The change of on-hand inventory

Inventory cost for perfect objects is

$$
I C=h_{p} \frac{b}{a}\left[(1-\lambda)\left(t_{1}-\frac{1}{a} e_{r}^{a t_{1}}\right)+\frac{1}{a}(2+\lambda)+\left\{2-e_{r}^{a t_{1}}-\lambda\left(1-e^{a t_{1}}\right)\right\} t^{\prime}{ }_{2}-\frac{1}{a} e_{r}^{a t_{2}^{\prime}}\right]
$$

Using Eq. (29), we get

$$
I C=h_{p} \frac{b}{a}\left[\begin{array}{l}
(1-\lambda)\left(t_{1}-\frac{1}{a} e_{r}^{a t_{1}}\right)+\frac{1}{a}(2+\lambda) \\
+\left\{1-e_{r}^{a t_{1}}-\lambda\left(1-e^{a t_{1}}\right)\right\} \frac{1}{a} \log \left[2-e_{r}^{a t_{1}}-\lambda\left(1-e^{a t_{1}}\right)\right]
\end{array}\right]
$$

Here, rework cost is zero.

Total expected profit

$$
=E\left[\int_{t_{1}}^{\infty}\left\{\begin{array}{l}
\left\{w_{p}+\overline{w_{s}} \frac{\beta}{1-\beta}\right\} b\left\{\lambda(1-\beta)-1+e_{r}^{a k T}\right\}-p_{I}\left\{(T-k T)+A_{p}\right\} \\
-h_{p} \frac{b}{a}\left\{\begin{array}{l}
(1-\lambda)\left(t_{1}-\frac{1}{a} e_{r}^{a t_{1}}\right)+\frac{1}{a}(2+\lambda) \\
+\left\{1-e_{r}^{a t_{1}}-\lambda\left(1-e^{a t_{1}}\right)\right\} \frac{1}{a} \log \left\{2-e_{r}^{a t_{1}}-\lambda\left(1-e^{a t_{1}}\right)\right\}
\end{array}\right\}
\end{array}\right] d F(\tau)\right]
$$

Adding Eq. (24) and Eq. (30), the total expected profit is 


$$
\begin{aligned}
& {\left[\left\{w_{p}+\frac{\overline{w_{s}} \beta}{1-\beta}\right\} b\left\{\lambda(1-\beta)-1+e_{r}^{a k T}\right\} \int_{0}^{t} d F(\tau)-p_{I}\left\{(T-k T)+A_{p}\right\} \int_{0}^{t} d F(\tau)\right.} \\
& -\left\{\begin{array}{l}
\frac{h_{p} b}{a^{2}}\left(e_{r}^{a t_{2}}-e_{r}^{a t_{1}}+e_{r}^{a t_{3}}-1+\lambda-\lambda e_{1}^{a t_{r}}\right) \\
+\frac{h_{p}{ }_{p} \lambda b}{a^{2}}\left(e_{1}^{a t_{r}}-a t_{r}-1\right)+\frac{h_{p} b}{a}\left(\lambda t_{r}-t_{3}\right)
\end{array}\right\} \\
& E P=E\left[\int_{0}^{t_{1}} d F(\tau)+\frac{h_{p} \lambda b}{a^{2}} \int_{0}^{t_{1}}\left(1-e^{a \tau}\right) d F(\tau)\right. \\
& -h_{p}\left(\frac{b(1-\beta) \lambda}{a^{2}}+\frac{\beta \lambda b t_{3}}{a}\right)_{0}^{t_{1}}\left(e^{a t_{1}}-e^{a \tau}\right) d F(\tau) \\
& -h_{p} \beta \lambda b e^{a t}\left(t_{2}-t_{r}\right) \int_{0}^{t_{1}}\left(t_{1}-\tau\right) d F(\tau)-h_{p} \frac{b}{a} \int_{0}^{t_{1}}\left(\lambda e^{a t_{1}}-e_{r}^{a t_{1}}-\lambda e^{a \tau}+e_{r}^{a \tau}\right) \tau d F(\tau)
\end{aligned}
$$

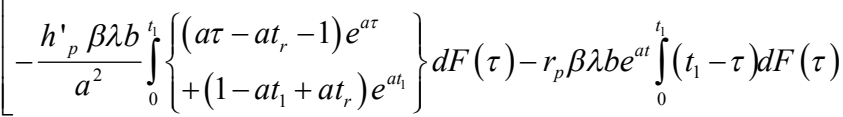

$$
\begin{aligned}
& +E\left[\int_{t_{1}}^{\infty}\left\{\begin{array}{l}
\left\{w_{p}+\overline{w_{s}} \frac{\beta}{1-\beta}\right\} b\left\{\lambda(1-\beta)-1+e_{r}^{a k T}\right\}-p_{I}\left\{(T-k T)+A_{p}\right\} \\
-h_{p} \frac{b}{a}\left\{\begin{array}{l}
(1-\lambda)\left(t_{1}-\frac{1}{a} e_{r}^{a t_{1}}\right)+\frac{1}{a}(2+\lambda) \\
+\left\{1-e_{r}^{a t_{1}}-\lambda\left(1-e^{a t_{1}}\right)\right\} \frac{1}{a} \log \left\{2-e_{r}^{a t_{1}}-\lambda\left(1-e^{a t_{1}}\right)\right\}
\end{array}\right\}
\end{array}\right] d F(\tau)\right]
\end{aligned}
$$

Thus, we have $\int_{0}^{t_{1}}\left(t_{1}-\tau\right)^{2} d F(\tau)=\theta \int_{0}^{t_{1}}\left(t_{1}-\tau\right)^{2} e^{-\theta \tau} d \tau=t_{1}^{2}-\frac{2 t_{1}}{\theta}+\frac{2}{\theta^{2}}\left(1-e^{-\theta t_{1}}\right)$. Expand the function $e^{-\theta t_{1}}$ up to fourth term, we have $\int_{0}^{t_{1}}\left(t_{1}-\tau\right)^{2} d F(\tau) \approx \frac{\theta t_{1}^{3}}{3}$. Thus, the average expected profit of the producer is

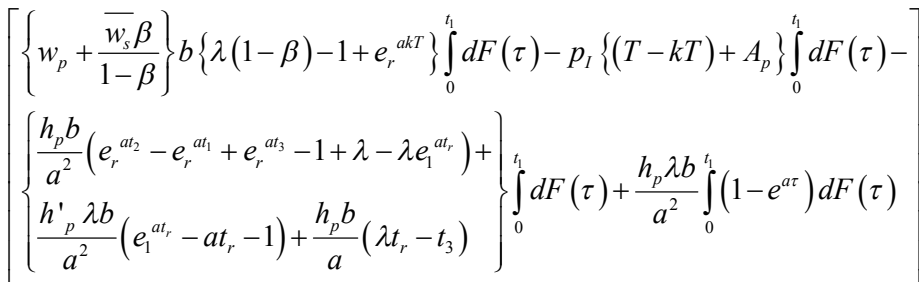

$$
\begin{aligned}
& E P A P=\frac{1}{T}-h_{p}\left(\frac{b(1-\beta) \lambda}{a^{2}}+\frac{\beta \lambda b t_{3}}{a} \int_{0}^{t_{1}}\left(e^{a t_{1}}-e^{a \tau}\right) d F(\tau)-r_{p} \beta \lambda b e^{a t_{1}} \int_{0}^{t_{1}}\left(t_{1}-\tau\right) d F(\tau)-\right.
\end{aligned}
$$

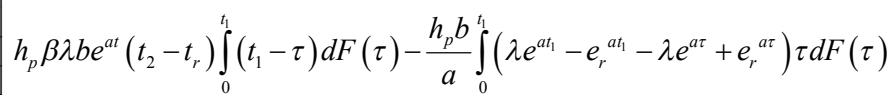

$$
\begin{aligned}
& -h_{p}^{\prime} \frac{\beta \lambda b}{a^{2}} \int_{0}^{t_{1}}\left(e^{a t_{1}}-e^{a \tau}+a \tau e^{a \tau}-a t_{1} e^{a t_{1}}+a t_{r} e^{a t_{1}}-a t_{r} e^{a \tau}\right) d F(\tau) \\
& +\frac{1}{T}\left[\int_{t_{1}}^{\infty}\left\{\begin{array}{l}
\left\{w_{p}+\overline{w_{s}} \frac{\beta}{1-\beta}\right\} b\left\{\lambda(1-\beta)-1+e_{r}^{a k T}\right\}-p_{I}\left\{(T-k T)+A_{p}\right\} \\
-h_{p} \frac{b}{a}\left\{\begin{array}{l}
(1-\lambda)\left(t_{1}-\frac{1}{a} e_{r}^{a_{1}}\right)+\frac{1}{a}(2+\lambda) \\
+\left\{1-e_{r}^{a t_{1}}-\lambda\left(1-e^{a t_{1}}\right)\right\} \frac{1}{a} \log \left[2-e_{r}^{a t_{1}}-\lambda\left(1-e^{a t_{1}}\right)\right]
\end{array}\right\}
\end{array}\right] d F(\tau)\right]
\end{aligned}
$$




\subsection{Retailer's specific average profit.}

The leading differential equations are

$\frac{d Q_{r}(t)}{d t}=b\left(e_{r}^{a t}-e_{c}^{a t}\right)$

with $Q_{r}(0)=0,0 \leq t \leq k T$

and

$\frac{d Q_{r}(t)}{d t}=-b e_{c}^{a t}$

with $Q_{r}(T)=0, k T \leq t \leq T$

From Eq. (33) and Eq. (34), we have

$Q_{r}(t)=\frac{b}{a}\left(e_{r}^{a t}-e_{c}^{a t}\right), 0 \leq t \leq k T$

and using $Q_{r}(k T)=\frac{b}{a}\left(e_{r}^{a k T}-e_{c}^{a k T}\right)$

$Q_{r}(t)=\frac{b}{a}\left(e_{r}^{a k T}-e_{c}^{a t}\right), k T \leq t \leq T$

Thus, $Q_{r}(T)=0 \Rightarrow k=\frac{b e_{c}^{a t}}{b e_{r}{ }^{a t}}<1$ as $e_{r}^{a t}>e_{c}^{a t}$

For possibility of the model, $t_{1} \leq k T<T$ must be gratified. As stated, $k T<T$ grasps as $k<1$.

Now, we have $t_{1} \leq k T$

$t_{1} \leq \frac{(1-\beta) \lambda b e^{a t} t_{1}}{b e_{r}^{a t}} \Rightarrow(1-\beta) \lambda e^{a t} \geq e_{r}^{a t} \Rightarrow \lambda E(1-\beta) e^{a t} \geq e_{r}^{a t}$

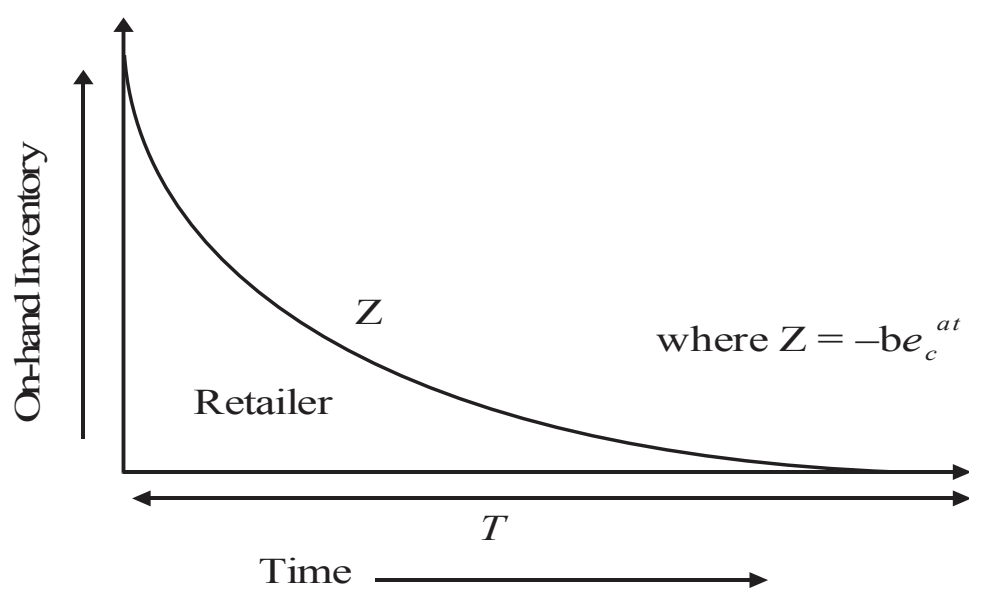

Fig. 5. The change of on-hand inventory 
The cost of arrangement is $A_{r}$

The income from selling items is $w_{r} b e_{c}^{a t} T$

The cost of buying objects is $w_{p} b e_{c}^{a t} T$.

The cost of inventory is

$$
H_{r}=h_{r}\left[\int_{0}^{k T} Q_{r}(t) d t+\int_{k T}^{T} Q_{r}(t) d t\right]=h_{r} \frac{b}{a^{2}}\left\{e_{c}^{a k T}-2 e_{c}^{a T}+e_{r}^{a k T}(1+a T-a k T)\right\}
$$

The retailer's average profit is

$$
R A P=\frac{1}{T}\left[w_{r} b e_{c}^{a t} T-A_{r}-w_{p} b e_{c}^{a t} T-h_{r} \frac{b}{a^{2}}\left\{e_{c}^{a k T}-2 e_{c}^{a T}+e_{r}^{a k T}(1+a T-a k T)\right\}\right]
$$

\subsection{Leader-Follower Association}

Producer is the leader; supplier and retailer are the followers.

From Eq. (32), using $t_{1}=\frac{1}{a} \log \left(1+\frac{a(1-\alpha) R}{\lambda b}\right), b e_{r}^{a k t}=b e_{c}^{a t}$ and $T=\frac{1}{a k} \log \left\{1+\frac{a}{b}(1-\alpha) R\right\}$

The producer's expected average profit is

$$
\begin{aligned}
& \mathrm{E}[\mathrm{PAP}]=\left[\frac{a k}{\log \left\{1+\frac{a}{b} E(1-\alpha) R\right\}}\right] \\
& {\left[\begin{array}{l}
\left\{\lambda b\left(w_{p}+\beta \overline{w_{p}}-S_{p}-C(P)-\frac{h_{p} \beta \lambda b}{r_{p}}\right)-A_{p}\right\}-\frac{h_{p} a^{2} \beta E\left((1-\alpha)^{2}\right)}{r_{p}} R^{2} \\
+\left\{\left(w_{p}-\frac{h_{p}}{a^{2}}\right)+\beta\left(\overline{w_{p}}-\frac{h_{p}}{a^{2}}-\frac{2 h_{p} \lambda b}{r_{p}}\right)-\left(S_{p}+C(P)\right)\right\} a E(1-\alpha) R \\
-\left(\frac{b h_{p}}{a^{2}}+\frac{h_{p}}{a} E(1-\alpha) R+\frac{p_{I}}{a k}\right) \log \left\{1+\frac{a}{b} E(1-\alpha) R\right\} \\
+\left(\frac{b h_{p}}{a^{2}} \lambda+\frac{h_{p}}{a} E(1-\alpha) R+\frac{h_{p} \beta \lambda b}{a^{2}}+\frac{p_{I}}{a}\right) \log \left(1+\frac{a E(1-\alpha) R}{\lambda b}\right)
\end{array}\right]}
\end{aligned}
$$

$E[P A P]=\left[\frac{P}{\log (1+N R)}\right]\left[M+K S R-H R^{2}-(J+L R+G) \log (1+N R)+(F+L R+D+B) \log (1+A R)\right]$

where $P=a k, N=\frac{a}{b} E(1-\alpha), D=\frac{h_{p} \beta \lambda b}{a^{2}}, B=\frac{p_{I}}{a}, A=\frac{a E(1-\alpha)}{\lambda b}, J=\frac{b h_{p}}{a^{2}}, F=\frac{b h_{p}}{a^{2}} \lambda$ 


$$
\begin{aligned}
& M=\left\{\lambda b\left(w_{p}+\beta \overline{w_{p}}-S_{p}-C(P)-\frac{h_{p} \beta \lambda b}{r_{p}}\right)-A_{p}\right\}, L=\frac{h_{p}}{a} E(1-\alpha), S=a E(1-\alpha) \\
& K=\left\{\left(w_{p}-\frac{h_{p}}{a^{2}}\right)+\beta\left(\overline{w_{p}}-\frac{h_{p}}{a^{2}}-\frac{2 h_{p} \lambda b}{r_{p}}\right)-\left(S_{p}+C(P)\right)\right\}, G=\frac{p_{I}}{a k}, H=\frac{h_{p} a^{2} \beta E\left((1-\alpha)^{2}\right)}{r_{p}}
\end{aligned}
$$

From Eq. (6), using $t_{1}=\frac{1}{a} \log \left(1+\frac{a(1-\alpha) R}{\lambda b}\right), b e_{r}^{a k t}=b e_{c}^{a t} \quad$ and $T=\frac{1}{a k} \log \left\{1+\frac{a}{b}(1-\alpha) R\right\}$

The supplier's expected average profit is

$$
E[S A P]=\left[\frac{a k}{\log \left\{1+\frac{a}{b} E(1-\alpha) R\right\}}\right]\left[\begin{array}{l}
E(1-\alpha) R w_{s}+E(\alpha) R \overline{w_{s}}-R\left(S_{c}+C_{s}\right)-E(1-\alpha) R \frac{h_{s}}{a}-A_{s} \\
+\frac{a E(\alpha) R^{2}}{r_{s}}-I_{s} \frac{1}{a k} \log \left\{1+\frac{a}{b} E(1-\alpha) R\right\} \\
-\left(\frac{\lambda b}{a} h_{s}+E(1-\alpha) R h_{s}-I_{s}\right) \frac{1}{a} \log \left(1+\frac{a E(1-\alpha) R}{\lambda b}\right)
\end{array}\right]
$$

From Eq. (39), using $t_{1}=\frac{1}{a} \log \left(1+\frac{a(1-\alpha) R}{\lambda b}\right), b e_{r}^{a k t}=b e_{c}^{a t}$ and $T=\frac{1}{a k} \log \left\{1+\frac{a}{b}(1-\alpha) R\right\}$

The retailer's expected average profit is

$$
E[R A P]=\left(w_{r}-w_{p}-\frac{h_{r}}{a}(1-k)\right)\{b+a E(1-\alpha) R\}-\frac{a k A_{r}}{\log \left\{1+\frac{a}{b} E(1-\alpha) R\right\}}
$$

Solution:

For optimum value of EPAP $[R]$, we have

$$
\begin{array}{r}
\frac{\partial E P A P}{\partial R}=\frac{P}{\log (1+N R)}\left[\begin{array}{l}
K S-2 H R-L \log (1+N R) \\
+(F+D+B+L R) \log A \\
+L \log (1+A R)-(J+L R+G) \log N
\end{array}\right] \\
-\frac{P N}{(\log (1+N R))^{2}}\left[\begin{array}{l}
M+K S R-H R^{2}+(F+D+B+L R) \\
\log (1+A R)-(J+L R+G) \log (1+N R)
\end{array}\right]
\end{array}
$$

We consider $\frac{\partial E P A P}{\partial R}=0$, which give us the value of $\mathrm{R}$

$$
R=\frac{(H+L \log N-L \log A)-\sqrt{\begin{array}{l}
(-H-L \log N+L \log A)^{2}-\left(-N H-L(\log N)^{2}+N L \log A\right) \\
(-N M+K G-F \log N-D \log N-B \log N \\
+K \log A+D \log A+N \log A)
\end{array}}}{\left(-N H-L(\log N)^{2}+N L \log A\right)}
$$

Again, we have the value of 


$$
\frac{\partial^{2} E P A P}{\partial R^{2}}=-2 \frac{P}{(\log (1+N R))^{3}}\left[\begin{array}{l}
\left(\begin{array}{l}
\log (1+N R))^{2}(H+L \log N-L \log A)+N \log (1+N R) \\
(L S-2 H R-L \log (1+N R)+(F+D+B+L R) \log A \\
+L \log (1+A R)-(J+L R+G) \log N
\end{array}\right) \\
+N^{2}\left(\begin{array}{l}
-M-K S R+H R^{2}-(K+D+B+L R) \log (1+A R) \\
+(J+L R+G) \log (1+N R)
\end{array}\right)
\end{array}\right]
$$

Thus $\frac{\partial^{2} E P A P}{\partial R^{2}}<0$ grasps at $R$, then $\operatorname{EPAP}[R] \quad$ is maximum.

\subsection{Integrated expected average profit}

The integrated expected average profit of supply chain is

$$
\begin{gathered}
\operatorname{EIAP}=b\left(w_{r}-w_{p}-\frac{h_{r}}{a}(1-k)\right)-I_{s}-p_{I}-\frac{b k h_{p}}{a}+a\left(w_{r}-w_{p}-\frac{h_{r}}{a}(1-k)-\frac{k h_{p}}{a}\right) E(1-\alpha) R \\
\left.\begin{array}{l}
\lambda b w_{p}+\lambda b \beta \overline{w_{p}}-\lambda b S_{p}-\lambda b C(P)-A_{p}-A_{s}-A_{r}-\frac{h_{p} \beta \lambda^{2} b^{2}}{r_{p}} \\
\log \left\{1+\frac{a}{b} E(1-\alpha) R\right\}
\end{array}\right]\left[\begin{array}{l}
+\left(\overline{w_{s}} E(\alpha)-S_{c}-C_{s}\right) R+a\left(\frac{E(\alpha)}{r_{s}}-\frac{h_{p} a \beta E\left((1-\alpha)^{2}\right)}{r_{p}}\right) R^{2} \\
+\left\{a w_{p}+w_{s}+a \beta \overline{w_{p}}-a S_{p}-a C(P)-\frac{h_{s}}{a}-\frac{h_{p}}{a}-\frac{\beta h_{p}}{a}-\frac{2 a \beta h_{p} \lambda b}{r_{p}}\right\} E(1-\alpha) R \\
+\left(p_{I}+I_{s}+\frac{\lambda b h_{p}}{a}+\frac{h_{p} \beta \lambda b}{a}-\frac{\lambda b h_{s}}{a}+\left(h_{p}-h_{s}\right) E(1-\alpha) R\right) \frac{1}{a} \log \left(1+\frac{a E(1-\alpha) R}{\lambda b}\right)
\end{array}\right] \\
E I A P=A_{1}+B_{1} C_{1} R_{1}+\frac{D_{1}}{\log \left(1+E_{1} C_{1} R_{1}\right)}\left[\begin{array}{l}
F_{1}+\left(G_{1}+J_{1} C_{1}\right) R_{1}+H_{1} R_{1}^{2} \\
+\left(K_{1}+L_{1} C_{1} R_{1}\right) M_{1} \log \left(1+N_{1} C_{1} R_{1}\right)
\end{array}\right]
\end{gathered}
$$

Where $A_{1}=b\left(w_{r}-w_{p}-\frac{h_{r}}{a}(1-k)\right)-I_{s}-p_{I}-\frac{b k h_{p}}{a}, \quad B_{1}=a\left(w_{r}-w_{p}-\frac{h_{r}}{a}(1-k)-\frac{k h_{p}}{a}\right)$

$$
\begin{aligned}
& C_{1}=E(1-\alpha), D_{1}=a k, E_{1}=\frac{a}{b}, F_{1}=\lambda b w_{p}+\lambda b \beta \overline{w_{p}}-\lambda b S_{p}-\lambda b C(P)-A_{p}-A_{s}-A_{r}-\frac{h_{p} \beta \lambda^{2} b^{2}}{r_{p}}, \\
& G_{1}=\overline{w_{s}} E(\alpha)-S_{c}-C_{s}, H_{1}=a\left(\frac{E(\alpha)}{r_{s}}-\frac{h_{p} a \beta E\left((1-\alpha)^{2}\right)}{r_{p}}\right), \\
& J_{1}=\left\{a w_{p}+w_{s}+a \beta \overline{w_{p}}-a S_{p}-a C(P)-\frac{h_{s}}{a}-\frac{h_{p}}{a}-\frac{\beta h_{p}}{a}-\frac{2 a \beta h_{p} \lambda b}{r_{p}}\right\}, \\
& K_{1}=p_{I}+I_{s}+\frac{\lambda b h_{p}}{a}+\frac{h_{p} \beta \lambda b}{a}-\frac{\lambda b h_{s}}{a}, L_{1}=h_{p}-h_{s}, M_{1}=\frac{1}{a}, N_{1}=\frac{a}{\lambda b}
\end{aligned}
$$

Solution: For optimum value of $E I A P\left[R_{1}\right]$, we have 


$$
\begin{aligned}
\frac{\partial E I A P}{\partial R_{1}}= & B_{1} C_{1}+\frac{D_{1}\left(G_{1}+C_{1} J_{1}+2 H_{1} R_{1}+C_{1} M_{1}\left(K_{1}+C_{1} L_{1} R_{1}\right) \log N_{1}+C_{1} L_{1} M_{1} \log \left(1+C_{1} N_{1} R_{1}\right)\right)}{\log \left(1+E_{1} C_{1} R_{1}\right)} \\
& -\frac{C_{1} D_{1} E_{1}\left(F_{1}+\left(G_{1}+C_{1} J_{1}\right) R_{1}+H_{1} R_{1}^{2}+M_{1}\left(K_{1}+C_{1} L_{1} R_{1}\right) \log \left(1+C_{1} N_{1} R_{1}\right)\right)}{\left(\log \left(1+E_{1} C_{1} R_{1}\right)\right)^{2}}
\end{aligned}
$$

Put $\frac{\partial E I A P}{\partial R_{1}}=0$, we have

$$
R_{1}=\frac{-\left(D_{1} H_{1}+B_{1} C_{1}^{2} \log E_{1}+C_{1}^{2} D_{1} L_{1} M_{1} \log N_{1}\right)+\sqrt{\begin{array}{l}
\left(D_{1} H_{1}+B_{1} C_{1}^{2} \log E_{1}+C_{1}^{2} D_{1} L_{1} M_{1} \log N_{1}\right)^{2} \\
\left.-\begin{array}{l}
-C_{1} D_{1} E_{1} F_{1}+D_{1} G_{1}+C_{1} D_{1} J_{1}+C_{1} D_{1} K_{1} M_{1} \log N_{1} \\
-C_{1} D_{1} K_{1} M_{1} \log E_{1}+\left(B_{1}+D_{1} L_{1} M_{1}\right) \log C_{1}
\end{array}\right) \\
\left(C_{1} D_{1} E_{1} H_{1}+B_{1} C_{1}^{3}\left(\log E_{1}\right)^{2}+C_{1}^{3} D_{1} E_{1} L_{1} M_{1} \log N_{1}\right)
\end{array}}}{\left(C_{1} D_{1} E_{1} H_{1}+B_{1} C_{1}^{3}\left(\log E_{1}\right)^{2}+C_{1}^{3} D_{1} E_{1} L_{1} M_{1} \log N_{1}\right)}
$$

Again partially differentiate, we have

$$
\frac{\partial^{2} E I A P}{\partial R_{1}^{2}}=-2 D_{1}\left[\begin{array}{l}
-\frac{H_{1}+C_{1}^{2} L_{1} M_{1} \log N_{1}}{\log \left(1+E_{1} C_{1} R_{1}\right)}+\frac{C_{1} E_{1}\left(\begin{array}{l}
G_{1}+C_{1} J_{1}+2 H_{1} R_{1}+C_{1} M_{1} \log N_{1}\left(K_{1}+C_{1} L_{1} R_{1}\right) \\
+C_{1} L_{1} M_{1} \log \left(1+C_{1} N_{1} R_{1}\right)
\end{array}\right)}{\left(\log \left(1+E_{1} C_{1} R_{1}\right)\right)^{2}} \\
-\frac{C_{1}^{2} E_{1}^{2}\left(F_{1}+\left(G_{1}+C_{1} J_{1}\right) R_{1}+H_{1} R_{1}^{2}+M_{1}\left(K_{1}+C_{1} L_{1} R_{1}\right) \log \left(1+C_{1} N_{1} R_{1}\right)\right)}{\left(\log \left(1+E_{1} C_{1} R_{1}\right)\right)^{3}}
\end{array}\right]
$$

Thus, $\frac{\partial^{2} E I A P}{\partial R_{1}^{2}}<0$ grasps at $R_{1}$, and then $E I A P\left[R_{1}\right]$ is maximum.

\section{Numerical Example}

The following parameters in applicable units are considered as:

$A_{s}=\$ 400, r_{s}=185,000$ units per unit time, $C_{s}=\$ 20$ per unit, $S_{c}=\$ 0.6$ per unit, $b=2$ per unit, $k=0.5$ per unit, $h_{s}=\$ 3.5$ per unit per unit time, $I_{s}=\$ 30$ per unit time, $a=0.7$ per unit, $w_{s}=\$ 70$ per unit, $\overline{w_{s}}=\$ 40$ per unit, $A_{p}=\$ 5000, r_{p}=180,000$ units per unit time, $S_{p}=\$ 0.8$ per unit, $h_{p}=\$ 4.5$ per unit per unit time, $p_{I}=\$ 20$ per unit time, $w_{p}=\$ 600$ per unit, $\overline{w_{p}}=\$ 400$ per unit, $b e_{c}^{a t}=300$ units, $A_{r}=\$ 4000, h_{r}=\$ 6$ per unit per unit time, $w_{r}=\$ 620$ per unit, $\gamma=\$ 0.02$ per unit, $\lambda=\$ 1.5$ per unit, $L=\$ 4500, f(\alpha)=\frac{1}{0.3-0.04}, 0.04<\alpha<0.3, \quad \beta=0.05$. The ideal outcome for integrated network is $R=\$ 110.5$ units, $E R A P=\$ 3776.7, E S A P=\$ 758.1, \quad E P A P=\$ 7638.1 . \quad$ Total profit of the supply chain is $\$ 12172.9$.

\section{Sensitivity Analysis}

To analysis, how the optimal solution is influenced by the parameters, we derive the sensitivity analysis for all parameters. From the given mathematical model, we obtain the optimum result for a stable segment. 
The optimum values of all parameters in the segment increases or decreases by $5 \%,-5 \%$ and $10 \%,-10 \%$. The results of total profit are presented in Table 1 as follows,

Table 1

The summary of sensitivity analysis

\begin{tabular}{|c|c|c|c|c|c|c|}
\hline Parameters & Values & $\begin{array}{c}\text { Values } \\
I_{s}=0 \text { and } p_{I}=0\end{array}$ & $\begin{array}{c}\text { Values } \\
+5 \%\end{array}$ & $\begin{array}{l}\text { Values } \\
+10 \%\end{array}$ & $\begin{array}{l}\text { Values } \\
-5 \%\end{array}$ & $\begin{array}{l}\text { Values } \\
-10 \%\end{array}$ \\
\hline$A_{s}$ & 400 & 400 & 420 & 440 & 380 & 360 \\
\hline$r_{s}$ & 185,000 & 185,000 & 194,250 & 203,500 & 175,750 & 166,500 \\
\hline$C_{s}$ & 20 & 20 & 21 & 22 & 19 & 18 \\
\hline$S_{c}$ & 0.6 & 0.6 & 0.63 & 0.66 & 0.57 & 0.54 \\
\hline$b$ & 2 & 2 & 2.1 & 2.2 & 1.9 & 1.8 \\
\hline$k$ & 0.5 & 0.5 & 0.53 & 0.55 & 0.47 & 0.45 \\
\hline$h_{s}$ & 3.5 & 3.5 & 3.68 & 3.85 & 3.32 & 3.15 \\
\hline$I_{s}$ & 30 & 0 & 31.5 & 33 & 28.5 & 27 \\
\hline$a$ & 0.7 & 0.7 & 0.74 & 0.77 & 0.66 & 0.63 \\
\hline$w_{s}$ & 70 & 70 & 73.5 & 77 & 66.5 & 63 \\
\hline $\bar{W}_{s}$ & 40 & 40 & 42 & 44 & 38 & 36 \\
\hline$A_{p}$ & 5000 & 5000 & 5250 & 5500 & 4750 & 4500 \\
\hline$r_{p}$ & 180000 & 180000 & 189000 & 198000 & 171000 & 162000 \\
\hline$S_{p}$ & 0.8 & 0.8 & 0.84 & 0.88 & 0.76 & 0.72 \\
\hline$h_{p}$ & 4.5 & 4.5 & 4.73 & 4.95 & 4.27 & 4.05 \\
\hline$p_{I}$ & 20 & 0 & 21 & 22 & 19 & 18 \\
\hline$w_{p}$ & 600 & 600 & 630 & 660 & 570 & 540 \\
\hline$\overline{w_{n}}$ & 400 & 400 & 420 & 440 & 380 & 360 \\
\hline$b e_{c}^{a t}$ & 300 & 300 & 315 & 330 & 285 & 270 \\
\hline$A_{r}$ & 4000 & 4000 & 4200 & 4400 & 3800 & 3600 \\
\hline$h_{r}$ & 6 & 6 & 6.3 & 6.6 & 5.7 & 5.4 \\
\hline$w_{r}$ & 620 & 620 & 651 & 682 & 589 & 558 \\
\hline$\gamma$ & 0.02 & 0.02 & 0.021 & 0.022 & 0.019 & 0.018 \\
\hline$\lambda$ & 1.5 & 1.5 & 1.58 & 1.65 & 1.42 & 1.35 \\
\hline $\mathrm{L}$ & 4500 & 4500 & 4725 & 4950 & 4275 & 4050 \\
\hline $\mathrm{E}(\alpha)$ & 0.17 & 0.17 & 0.18 & 0.19 & 0.16 & 0.15 \\
\hline$\beta$ & 0.175 & 0.175 & 0.18 & 0.19 & 0.17 & 0.16 \\
\hline $\begin{array}{l}E(1-\alpha) \\
E(1-\alpha)^{2}\end{array}$ & $\begin{array}{c}0.83 \\
0.7\end{array}$ & $\begin{array}{c}0.83 \\
0.7\end{array}$ & $\begin{array}{l}0.83 \\
0.74\end{array}$ & $\begin{array}{l}0.91 \\
0.77\end{array}$ & $\begin{array}{l}0.79 \\
0.66\end{array}$ & $\begin{array}{l}0.75 \\
0.63\end{array}$ \\
\hline$R$ & 110.5 & 14.6 & 108.48 & 119.89 & 97.35 & 99.89 \\
\hline ESAP & 758.1 & 773.8 & 1051.48 & 1476.02 & 359.34 & 280.57 \\
\hline EPAP & 7638.1 & 7648.6 & 9708.03 & 23173.79 & 1558.17 & -22.91 \\
\hline ERAP & 3776.7 & 3776.7 & 4300.53 & 4977.69 & 3085.56 & 2721.49 \\
\hline Total Profit & 12172.9 & 12199.1 & 15060.04 & 29627.5 & 5003.07 & 2979.15 \\
\hline
\end{tabular}




\section{Conclusion}

The main conclusion draws from the sensitivity analysis is as follows: If the percentage of imperfect items $\beta$ increases in the out-of-control state and the value of reliability parameter $\theta$ increases then the optimal ordering size decreases and production rate increases. Hence, the expected average integrated profit decreases. The demand rate has impact on the three layers of the supply chain. The production rate has impact on the profit of producer. The production rate is demand dependent i.e. more sensitive to increases or decreases total profit of the supply chain. The values of other parameters are less sensitive to total profit of the supply chain.

In this paper, we considered three layer supply chain including supplier, producer and retailer. For mathematical model, we considered the demand rate of supplier and retailer was exponential increasing function of time. In addition, the production rate was demand dependent. The supplier supplies the material according to the demand of retailer. We have assumed to have constant product reliability; the cycle time at each stage is equal and stock-out situation at each level is insignificant.

Future research can be accomplished for including stock-out situation, shortages are allowed, multisupplier and multi-retailer may be considered.

\section{Acknowledgments}

The authors express the best wishes to the editors and referees for their guidance to improve the quality of the present paper.

\section{References}

Banerjee, A., \& Kim, S. L. (1995). An integrated JIT inventory model. International Journal of Operations and Production Management, 15, 237-244.

Biswas, P., \& Sarker, R.B. (2008). Optimal batch quantity models for a lean production system with incycle rework and scrap. International Journal of Production Research, 46, 6585-6610.

Cardenas, E.L., \& Barron. (2007). Optimizing inventory decisions in a multi-stage multi-customer supply chain. Transportation Research, 43, 647-654.

Cardenas, E.L., \& Barron. (2008). Optimal manufacturing batch size with rework in a single stage production system. Computers and Industrial Engineering, 55, 758-765.

Cardenas, E.L., \& Barron. (2009b). Economic production quantity with rework process at a single stage manufacturing system with planned backorders. Computers and Industrial Engineering, 57, 11051113.

Chang, H. H., \& Chend, T. F. (2010). Economic production quantity model with backordering, rework and machine failure taking place in stock piling time. Wseas Transactions on Information Science and Applications, 7, 463-473.

Chiu, S. W., Ting, C. K., \& Chiu, Y. S. P. (2007). Optimal production lot sizing with rework, scrap rate, and service level constraint. Mathematical and Computer Modelling, 46(3), 535-549.

Chiu, S. W., Wang, S. L., \& Chiu, Y. S. P. (2007). Determining the optimal run time for EPQ model with scrap, rework, and stochastic breakdowns. European Journal of Operational Research, 180(2), 664-676.

Chiu, P.Y. (2003). Determining the optimal lot size for the finite production model with random defective rate, the rework process and backlogging. Engineering Optimization, 5, 427-437.

Chiu, Y. S. P., Chiu, S. W., \& Chao, H. C. (2006). Numerical method for determination of reworking or scraping the defective items in a finite production rate model. Communications in numerical methods in engineering, 22(5), 377-386. 
Peter Chiu, Y. S., Chen, K. K., Cheng, F. T., \& Wu, M. F. (2010). Optimization of the finite production rate model with scrap, rework and stochastic machine breakdown. Computers \& mathematics with applications, 59(2), 919-932.

Chung, J.C., \& Wee, M. H. (2010). Green-product-design and information-technology investment on replenishment model with remanufacturing. International Journal of Computer Integrated Manufacturing, 23, 466-485.

Hafshejani, K. F., Valmohammadi, C., \& Khakpoor, A. (2012). Retracted: Using genetic algorithm approach to solve a multi-product EPQ model with defective items, rework, and constrained space. Journal of Industrial Engineering International, 8(1), 1-8.

Haji, B., Haji, R., \& Haji, A. (2009). Optimal batch production with rework and non-zero setup cost for rework. International Conference on Computers and Industrial Engineering, 857-862.

Jamal, A. M. M., Sarker, B. R., \& Mondal, S. (2004). Optimal manufacturing batch size with rework process at a single-stage production system. Computers \& Industrial Engineering, 47(1), 77-89.

Jalbar, B., Gutiérrez, J. M., \& Sicilia, J. (2008). Policies for a single-vendor multi-buyer system with finite production rate. Decision Support Systems, 46(1), 84-100.

Khouja, M., \& Mehrez, A. (1994). An economic production lot size model with imperfect quality and variable production rate. Journal of the Operational Research Society, 45, 1405-1417.

Kim, H.C., \& Hong, Y. (1999). An optimal run length in deteriorating production processes. International Journal of Production Economics, 58, 183-189.

Krishnamoorthi, C., \& Panayappan, S. (2013). An EPQ model for an imperfect production system with rework and shortages. International Journal of Operations Research, 17, 104-124.

Lee, L. H., \& Rosenblatt, J.M. (1987). Simultaneous determination of production cycle and inspection schedules in a production system. Management Sciences, 3, 1125-1136.

Liao, I.G., et al. (2009). Optimal economic production quantity policy for imperfect process with imperfect repair and maintenance. European Journal of Operational Research, 195,348-357.

Liu, J.J., \&Yang, P. (1996). Optimal lot-sizing in an imperfect production system with homogeneous reworkable jobs. European Journal of Operational Research, 91, 517-527.

Pasandideh, S. H. R., Niaki, S. T. A., \& Mirhosseyni, S. S. (2010). A parameter-tuned genetic algorithm to solve multi-product economic production quantity model with defective items, rework, and constrained space. The International Journal of Advanced Manufacturing Technology, 49(5-8), 827-837.

Rosenblatt, J.M., \& Lee, L.H. (1986). Economic production cycles with imperfect production processes. IIE Transactions, 18, 48-55.

Sarkar, B., Sana, S. S., \& Chaudhuri, K. (2010). Optimal reliability, production lot size and safety stock in an imperfect production system. International Journal of Mathematics in Operational Research, 2(4), 467-490.

Sarker, B. R., Jamal, A. M. M., \& Mondal, S. (2008). Optimal batch sizing in a multi-stage production system with rework consideration. European Journal of Operational Research, 184(3), 915-929.

Sana, S. S., Goyal, S. K., \& Chaudhuri, K. (2007). On a volume flexible inventory model for items with an imperfect production system. International Journal of Operational Research, 2(1), 64-80.

Sana, S. S., Goyal, S. K., \& Chaudhuri, K. (2007a). An imperfect production process in a volume flexible inventory model. International Journal of Production Economics, 105(2), 548-559.

Sana, S.S., \& Chaudhuri, S.K. (2010). An EMQ model in an imperfect production process. International Journal of Systems Science, 41, 635-646.

Sana, S.S. (2010). A production inventory model in an imperfect production process. European Journal of Operational Research, 200, 451-464.

Sana, S.S. (2010a). An economic production lot size model in an imperfect production system. European Journal of Operational Research, 201, 158-170.

Sana, S.S. (2011). A production inventory model of imperfect quality products in a three layer supply chain. Decision Support Systems, 50, 539-547.

Pal, B., Sana, S. S., \& Chaudhuri, K. (2012). Three-layer supply chain-a production-inventory model for reworkable items. Applied Mathematics and Computation, 219(2), 530-543. 
542

Wee, M.H., \& Chung, J.C. (2009). Optimizing replenishment policy for an integrated production inventory deteriorating model considering green component-value design and remanufacturing. International Journal of Production Research, 47(5), 1343-1368.

Yang, P. C., \& Wee, M. H. (2001). An arborescent inventory model in a supply chain system. Production Planning and Control, 12, 728-735. 